\title{
Frauen interessieren sich nicht nur für Hausarztmedizin
}

\author{
Nora Bienz \\ Dr. med., FMH Allgemeine Innere Medizin, Präsidentin VSAO Bern, Mitglied Geschäftsausschuss VSAO Schweiz, Spiez
}

Es gibt Aussagen, die man sich nicht lange auf der Zunge zergehen lassen muss, um ihren bitteren Gehalt zu spüren. "[...] Frauen interessieren sich im Unterschied zu ihren männlichen Kollegen vor allem für die Kinder- und Hausarztmedizin [...] In operativen Fächern wie beispielsweise der Orthopädie gibt es nur 10 Prozent Frauen, das interessiert sie weniger» [1]. Bitter daran ist vor allem, dass ganz andere Gründe als das mangelnde Interesse der Frauen zu dieser unbefriedigenden Situation führen.

Ärztinnen sind seltener in Fachbereichen mit einer operativen Tätigkeit zu finden. Dieser Teil der Aussage von FMH-Präsident Jürg Schlup in der TV-Sendung «10 vor 10» vom 29. Oktober 2018 stimmt. Was hingegen nicht stimmt, ist Schlups Begründung, Frauen würden sich weniger für operative Tätigkeiten interessieren. Die Gründe für die Wahl der Fachrichtung sind vielschichtig. Anschauungsunterricht bietet ein Blick zurück auf den Anfang meiner eigenen Berufskarriere.

\section{Von der Euphorie zur Ernüchterung}

Mein Staatsexamen legte ich 2011 an der Universität Bern ab. Mich interessierte vor allem die operative Gynäkologie. Darauf hatte ich bereits mein Wahlstudienjahr ausgelegt. Die erste Stelle wählte ich in der Viszeralchirurgie eines mittelgrossen Spitals. Ich arbeitete ausgesprochen gerne und entwickelte eine grosse Leidenschaft für die Chirurgie. Selbst nachts noch am Operationstisch zu stehen war faszinierend. 24 Stunden durchzuarbeiten empfand ich als Herausforderung im positiven Sinn. Wochenenddienste direkt zwischen zwei normalen Arbeitswochen betrachtete ich als notwendiges Übel, um mehr operieren zu können. Doch innerhalb eines Jahres büsste ich viel von der Anfangseuphorie ein. Ich sehnte mich nach mehr Zeit und Energie für mein Privatleben und meine ausserberuflichen Interessen.

\section{Schicht statt 12-Tage-Wochen}

Beim Bewerbungsgespräch für meine Anschlussstelle in der Gynäkologie wurde ich unter anderem gefragt, ob ich denn bereit sei, privat zurückzustecken. Spätestens da begann ich mir Gedanken über meine Zukunft zu machen und fragte mich, ob eine chirurgische Karriere wirklich erstrebenswert ist. Vorbilder in leitenden chirurgischen Positionen hatte ich damals keine. Auch sah ich keine Perspektive auf Besserung der Arbeitsbedingungen. Und ich wusste, dass ich irgendwann eine Familie haben wollte.

Weil ich die Gynäkologiestelle nicht nahtlos erhielt, bot sich mir die Möglichkeit, für sechs Monate auf die Intensivstation zu rotieren. Nach dem chirurgischen Jahr mit 12-Tage-Diensten, etlichen Pikettdiensten sowie knapp 400 Stunden Überzeit erlebte ich das tadellos organisierte Schichtsystem der Intensivstation mit einer gesetzeskonformen 50-Stunden-Woche als erholsam. Und so entschied ich mich nach etlichen Gesprächen und reiflicher Überlegung schliesslich gegen die operative Gynäkologie. Ich verlängerte den Vertrag auf der Intensivstation und organisierte mir eine Anschluss-Anstellung an einer A-Klinik für Innere Medizin. Hier fand ich einen relativ geregelten Arbeitsalltag, eine flache Hierarchie, eine hervorragende Weiterbildungs- und Förderungsstruktur und eine gute Team-Zusammenarbeit. 2017 erwarb ich schliesslich den Facharzttitel für Allgemeine Innere Medizin und sammelte erste Erfahrungen als Oberärztin. Mittlerweile befinde ich mich in der Zweitausbildung zur Intensivmedizinerin. Meine Entscheidung bereut habe ich bislang nicht.

\section{Frauen in der Sackgasse}

Viele meiner ehemaligen Kommilitoninnen sind heute Fachärztinnen oder stehen kurz vor dem Abschluss 


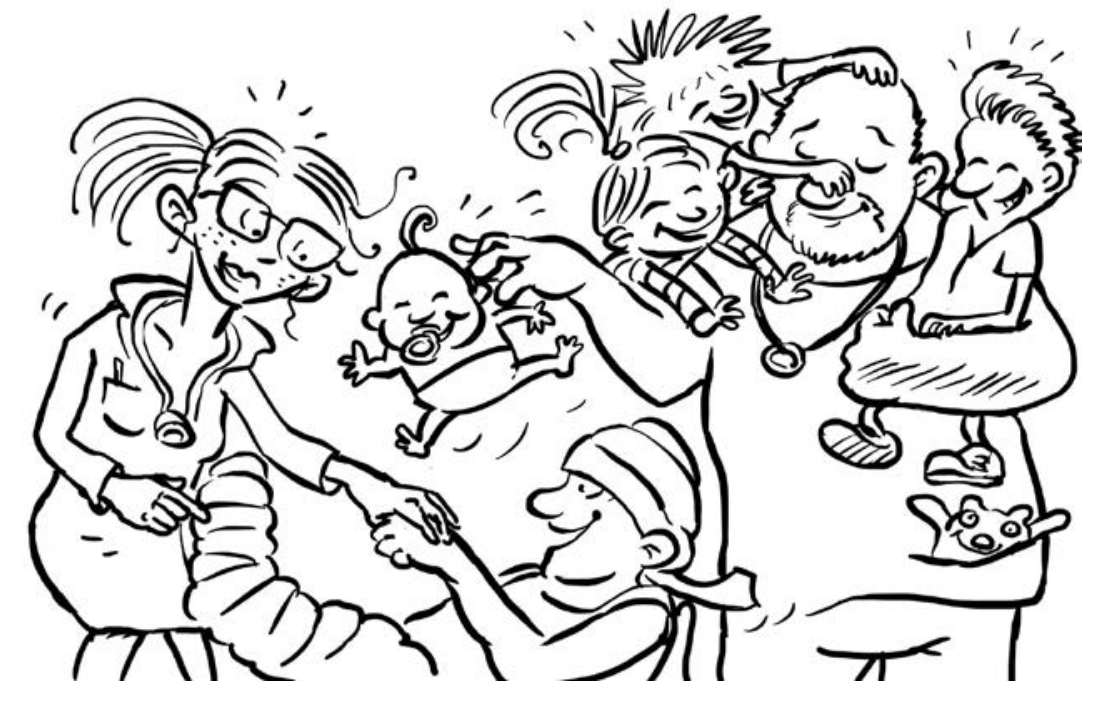

ihrer Weiterbildung. Einige wenige von ihnen sind der chirurgischen Laufbahn treu geblieben. Leider häufen sich in meinem Umfeld die Fälle von Ärztinnen, die trotz grossem Engagement, Begabung und Leidenschaft für ihren Beruf und (beinahe) abgeschlossener Weiterbildung nicht weiterkommen.

Die jungen Fachärztinnen werden in den Spitälern ungenügend gefördert oder nicht befördert und schlagen in der Not einen anderen Weg ein. Gerade Frauen in den Dreissigern - also im Zeitraum der Familienplanung - haben es besonders schwer, ihre Karriereziele zu verfolgen und umzusetzen. Das schlägt sich auch in den Zahlen nieder: Gemäss jüngster Datenerhebung des Regierungsrates des Kantons Bern [2] sind am Inselspital, dem grössten Arbeitgeber im Spitalsektor des Kantons, gerade mal 14 Prozent der Kaderstellen mit Ärztinnen besetzt.

Woran liegt es, dass sich Frauen bevorzugt in der Pädiatrie und der Inneren Medizin niederlassen? Und gibt es Lösungsvorschläge, damit Frauen auch vermehrt Kaderpositionen in Kliniken besetzen?

\section{Mehr Teilzeitarbeit - mehr Familie}

Wer die Ursachen dieser Entwicklung kennen will, muss nicht weit suchen: Es sind offensichtlich zwei gewichtige Faktoren, welche die Wahl des Fachgebiets wie auch die Karriereambitionen beeinflussen - die Möglichkeit zur Teilzeitarbeit und das Angebot an familienfreundlichen Arbeitsplätzen. Denn keine Frage: Die Vereinbarkeit von Beruf und Familie ist das zentrale Thema, wenn es um die beruflichen Perspektiven von Ärztinnen geht.

Dem trägt auch das jüngste Projekt des VSAO-Dachverbands Rechnung. Es setzt sich zum Ziel, die Teilzeit- arbeit an Schweizer Spitälern zu fördern. Nach einer Umfrage und Bedürfnisabklärung in verschiedenen Schweizer Spitälern liegt nun ein "Bauplan Teilzeit» vor. Damit sollen ab diesem Jahr massgeschneiderte Lösungen für Kliniken entstehen. Mit Hilfe solcher Lösungen wird sich die Klinikorganisation so anpassen lassen, dass (mehr) Teilzeitstellen realisierbar sind.

\section{Arbeitszeiten: 50 Stunden plus ...}

Es gibt weitere Gründe für den tiefen Frauenanteil in operativen Fächern und in Kaderpositionen. Sie sind z.T. ähnlich wie in anderen Branchen: die klassische Rollenverteilung in Schweizer Familien, die fehlende Bereitschaft von Unternehmen, Mutterschaftsurlaube in Kauf zu nehmen, aber auch die von Männern dominierten Unternehmensstrukturen und Netzwerke. Allerdings gibt es zusätzlich hausgemachte, das heisst ärztespezifische Faktoren. Dazu gehören

- die Spitalstrukturen mit ihren Arbeitsbedingungen,

- der enorm hohe Zeitaufwand für die nicht-ärztlichen Tätigkeiten,

- die hohe Sollarbeitszeit verbunden mit der Nichteinhaltung des Arbeitsgesetzes.

Die wöchentliche Arbeitszeit der Assistenzärzte is eine Besonderheit der ärztlichen Weiterbildung in der Schweiz. Im Vergleich zu den umliegenden Ländern ist sie höher und beträgt 50 Stunden pro Woche. Es liegt auf der Hand, dass bei einer solchen Arbeitszeit selbst ein 80-Prozent-Teilzeitpensum (entsprechend einer 40-Stunden-Woche) für eine Familie immer noch eine erhebliche Belastung darstellt. Gerade auch vor dem Hintergrund, dass jede(r) Zweite unter den Assistenzärzten in der Realität Woche für Woche länger als die gesetzlich zulässigen 50 Stunden im Dienst steht, mit allen negativen Konsequenzen für das eigene Befinden, das Wohl der Familie - und nicht zuletzt für die Patientensicherheit.

\section{Weniger Bürokratie, mehr ans Krankenbett}

Es mag auf den ersten Blick kein besonders origineller Gedanke sein: Doch gerade in den Spitälern lohnt es sich angesichts dieser grossen Arbeitsbelastung, mit der Verschlankung der bürokratischen Abläufe vorwärts zu machen. Denn Spitalärztinnen und -ärzte können heute gemäss einer aktuellen Studie der Universität Lausanne [3] gerade noch ein Drittel ihrer Arbeitszeit direkt am Krankenbett einsetzen. Unter dem Titel «Medizin statt Bürokratie!» versucht der 
VSAO mit einer Kampagne, Gegensteuer zu geben (www.medizin-statt-buerokratie.ch). Erfolgreich umgesetzte Beispiele zeigen, dass es Sinn macht, bestimmte administrative Aufgaben an speziell dafür ausgebildete Berufsgruppen zu delegieren oder zumindest zu vereinfachen.

Die Reduktion der ärztlichen Bürotätigkeit kommt - so die Überlegung - einerseits den Patienten zugute, andererseits trägt sie dazu bei, die Präsenzzeit im Spital effektiver zu nutzen. Weniger Administration kann mehr Operations- und Ausbildungszeit ermöglichen. Gerade eine chirurgische Tätigkeit erfordert nämlich viel Übung. Die erwähnte Lausanner Studie [3] besagt, dass nur gerade sechs Prozent der Arbeitszeit für die Ausbildung zur Verfügung stehen. Mehr Zeit im Spital bei so wenig Ausbildungszeit ist also nicht das Erfolgsrezept, um eine Topärztin zu werden. Ein Abbau der Bürokratie ist dabei wesentlich effektiver als die nächtliche Ausbildung übermüdeter Ärzte auf Überzeit.

\section{Vom Fördern und Fordern}

Die wissenschaftliche Arbeit bzw. Förderung ist eine weitere Voraussetzung, um in der Medizin eine Kaderposition zu erreichen. Wegen der hohen klinischen Arbeitsbelastung wird diese nicht selten als Freizeitforschung eingefordert, was unabhängig vom Geschlecht familienunverträglich ist. Auch was die Qualität der Forschung angeht, ist dieser Ansatz mehr als fragwürdig. Nur schon der enorme Aufwand bei der Beantragung von Forschungsgeldern ruft dringend nach mehr Freistellung von klinischen Arbeiten zumindest bei Ärztinnen mit Karriereambitionen. Zudem müssen wir uns die Frage stellen, ob Forschung und Klinik nicht stärker getrennt werden können. Wir brauchen die guten klinisch tätigen Ärztinnen nämlich genauso wie die guten Forscherinnen. Aber müssen sie für eine Kaderposition wirklich in beidem gut sein?

\section{Das Problem der Chefärzte}

An dieser Stelle muss schliesslich auch auf eine pikante Besonderheit im Spitalwesen hingewiesen werden. Ich spreche von den hierarchischen Strukturen, welche eine starke Abhängigkeit der angehenden Ärztinnen und Ärzte zur Folge hat. Gerade Entscheidungen betreffend Forschungsfreistellungen oder die Gewährung einer Fellow-Stelle in einer Partnerklinik im Ausland werden oft von Einzelpersonen in der Führungsebene gefällt.

Lehnt der/die Vorgesetzte eine Person ab, ist ein Weiterkommen oft schwierig. Und hält der Chef moderne
Teilzeitmodelle für nicht umsetzbar, ist ein klinischwissenschaftlich kombinierter Werdegang bei gleichzeitigen familiären Pflichten nur schwer realisierbar. Besonders in chirurgischen Fächern entscheidet die direkte Förderung durch die Vorgesetzte auch darüber, wie schnell ein Eingriffskatalog voll wird. Hinzu kommt, dass die Spitallandschaft in der Schweiz gerade im Bereich der hochspezialisierten Medizin relativ klein ist. Eine Ablehnung durch den einen Chef erschwert nicht selten auch in einem anderen Spital das Vorankommen. Eine faire Förderungs- und Beförderungskultur benötigt daher breit abgestützte Entscheidungsinstanzen. Letztere sollten zur Entscheidungsfindung objektiv überprüfbare Kriterien wie Fähigkeitsausweise, Sprachkenntnisse, Berufserfahrung, wissenschaftliche Publikationen, aber auch weiche Faktoren wie Sozialkompetenz und Teamfähigkeit heranziehen.

\section{Es braucht einen Kulturwandel}

Aktuell beträgt der Frauenanteil bei den Staatsexamen rund 60 Prozent, Tendenz steigend. Es ist also höchste Zeit, sich Gedanken darüber zu machen, welche längerfristigen Perspektiven diese Frauen im Beruf haben sollen. Das frühzeitige Ausscheiden von Ärztinnen aus dem aktiven Berufsleben verstärkt den bestehenden Fachkräftemangel. Wir möchten den teuer und gut ausgebildeten weiblichen Nachwuchs jedoch nicht nur im Beruf halten. Wir wollen, dass Ärztinnen Karriere machen können. Die Wahl des Fachs oder die Gründung einer Familie darf dabei kein Hindernis darstellen.

Flexible und familienfreundliche Arbeitsbedingungen für Frauen und Männer, eine Anpassung der klinikinternen Strukturen und ein grundlegender Kulturwandel sind unabdingbare Voraussetzungen, damit auch Ärztinnen die Fächerwahl unvoreingenommen und gemäss ihren tatsächlichen Interessen treffen können. Da wollen wir hin.

\section{Bildnachweis}

Nicolas d'Aujourd'hui

Literatur

1 Sendung 10vor10, 29. Oktober 2018, https://www.srf.ch/play/ tv/10vor10/video/10vor10-vom-29-10-2018?id=2f0198e2-ac24 4e9f-9ca0-5fof9d52e385.

2 Parlamentarischer Vorstoss, Antwort des Regierungsrates des Kantons Bern, Handlungsbedarf nach Diskriminierungsklage gegen Berner Inselspital: Gleichstellung von Frau und Mann in öffentlichen und privaten Spitälern und Kliniken des Kantons Bern verbessern! Geschäfts-Nr. 2018.RRGR.190.

3 Wenger N, Méan M, Castioni J, Marques-Vidal P, Waeber G, Garnier A. Allocation of Internal Medicine Resident Time in a Swiss Hospital: A Time and Motion Study of Day and Evening Shifts. Annals.org; 2017. 